\title{
Reflexos da amamentação na saúde bucal de bebês e na realidade maternal: revisão narrativa
}

\author{
Reflections of breastfeeding on infant oral health and maternal reality: a narrative review \\ Reflejos de la lactancia materna en la salud oral del lactante y la realidad materna: una revisión \\ narrativa
}

Recebido: 18/10/2021 | Revisado: 23/10/2021 | Aceito: 27/10/2021 | Publicado: 31/10/2021

\author{
Geovana Pereira \\ ORCID: https://orcid.org/0000-0003-3742-3501 \\ Centro Universitário de Patos de Minas, Brasil \\ E-mail: geovana@unipam.edu.br \\ Gabrielle Soares Campos \\ ORCID: https://orcid.org/0000-0002-7722-0250 \\ Centro Universitário de Patos de Minas, Brasil \\ E-mail: gabriellesc@unipam.edu.br \\ Carolina Silva Pereira \\ ORCID: https://orcid.org/0000-0003-2020-3932 \\ Centro Universitário de Patos de Minas, Brasil \\ E-mail:carolinasp@unipam.edu.br \\ Dayviddy Lucas Magalhães Silva \\ ORCID: https://orcid.org/0000-0002-6708-2659 \\ Centro Universitário de Patos de Minas, Brasil \\ E-mail:dayviddylms@unipam.edu.br \\ Laura Cesário Oliveira \\ ORCID: https://orcid.org/0000-0002-7689-5061 \\ Centro Universitário de Patos de Minas, Brasil \\ E-mail:lauracesario@unipam.edu.br \\ Thiago de Amorim Carvalho \\ ORCID: https://orcid.org/0000-0003-1153-0931 \\ Centro Universitário de Patos de Minas, Brasil \\ E-mail:thiagocarvalho@unipam.edu.br \\ Fabrício Campos Machado \\ ORCID: https://orcid.org/0000-0003-4603-8795 \\ Centro Universitário de Patos de Minas, Brasil \\ E-mail:fabriciocampos@unipam.edu.br
}

\begin{abstract}
Resumo
O aleitamento materno é a mais sábia estratégia natural de vínculo, afeto, proteção e nutrição para a criança e a mãe, e essa prática tem papel chave no desenvolvimento saudável acarretando em inúmeros benefícios. Nesse contexto, a Organização Mundial da Saúde recomenda a amamentação exclusiva por aproximadamente 6 meses, quando esse período de seis meses não é obedecido, a criança desenvolve maiores probabilidades de adquirir alterações funcionais. Baseado nisso, o presente artigo propõe, através de uma análise da literatura existente, informações esclarecedoras sobre a importância e necessidade da amamentação e os reflexos da mesma no sistema estomatognático da criança, os seus benefícios na saúde geral do binômio mãe-bebê, e as desvantagens na ausência dessa prática, sendo o desmame precoce um empecilho na vida do indivíduo e consequentemente há uma instalação de hábitos deletérios, e com isso, a privação do aleitamento materno posteriormente pode trazer problemas como desenvolvimento de oclusopatias, alterações do crescimento ósseo, respiração bucal, e funções anormais da língua durante a deglutição, fonação e postura.
\end{abstract}

Palavras-chave: Aleitamento materno; Criança; Desmame precoce; Comportamento de sucção; Saúde bucal.

\begin{abstract}
Breastfeeding is the wisest natural bonding, affection, protection and nutrition strategy for the child and the mother, and this practice has a key role in healthy development, bringing countless benefits. In this context, the World Health Organization recommends exclusive breastfeeding for approximately 6 months, when this period of six months is not obeyed, the child is more likely to acquire functional alterations. Based on this, this article proposes, through an analysis of the existing literature, clarifying information on the importance and need for breastfeeding and its effects on the child's stomatognathic system, its benefits on the general health of the mother-infant binomial, and the disadvantages in the absence of this practice, with early weaning being an obstacle in the individual's life and
\end{abstract}


consequently there is an installation of harmful habits, and with that, the deprivation of breastfeeding later can bring problems such as malocclusion development, changes in bone growth, breathing mouth, and abnormal tongue functions during swallowing, phonation and posture.

Keywords: Breastfeeding; Kid; Early weaning; Sucking behavior; Oral health.

\section{Resumen}

La lactancia materna es la estrategia natural más sabia de vinculación, afecto, protección y nutrición para el niño y la madre, y esta práctica tiene un papel clave en el desarrollo saludable, aportando innumerables beneficios. En este contexto, la Organización Mundial de la Salud recomienda la lactancia materna exclusiva durante aproximadamente 6 meses, cuando no se obedece este período de seis meses, es más probable que el niño adquiera alteraciones funcionales.Con base enesto, este artículo propone, mediante unanálisis de la literatura existente, aclarar información sobre la importancia y necesidad de la lactancia materna y sus efectos sobre el sistema estomatognático del niño, sus beneficios sobre la salud general del binomio madre-hijo y las desventajas em ausencia de esta práctica, siendo el destete precozun estorbo en la vida del individuo y en consecuencia se produce una instalación de hábitos nocivos, y conello, laprivación de lalactancia materna posterior puede traer problemas como eldesarrollo de maloclusiones, cambios em el crecimiento óseo, respiración boca y funciones anormales de la lengua durante la deglución, la fonación y la postura.

Palabras clave: Lactancia materna; Niño; Destete precoz; Conducta em la lactancia; Salud bucal.

\section{Introdução}

A prática odontológica envolve um conjunto de atividades e interfaces que interagem não somente com o processo saúde-doença bucal, mas também com os aspectos e impactos na saúde geral de um indivíduo. Diversas são condições patológicas bucais que acarretam consequências negativas na qualidade de vida dos indivíduos e um efeito nocivo na sociedade em que estão inseridos. Desse modo, o cuidado com a saúde bucal deve ser efetuado desde a primeira infância e perseverar durante a adolescência, vida adulta e na senescência, já que em todas essas etapas o ser humano está sujeito às desordens de sua saúde geral, física e psicossocial resultantes de uma saúde bucal debilitada (Bendo et al., 2014).

A odontopediatria objetiva contribuir na formação de uma geração com condições bucais menos problemáticas, com maior qualidade de vida e mais consciente acerca da importância das ações preventivas. A primeira infância caracteriza-se como um período crítico para o desenvolvimento do sistema estomatognático e oportuno para introdução de bons hábitos e padrões de comportamento que possam perdurar ao longo da vida. Dessa maneira, o exercício da odontologia infantil deve ter início durante o período gestacional com o intuito de instaurar o processo preventivo de doenças bucais (Fernandes et al., 2010).

Durante o curso da gestação alterações físicas, hormonais, e psicológicas estão presentes e o corpo da mulher passa por diversas transformações tocantes ao seu funcionamento. Nesse contexto, a necessidade de um acompanhamento multidisciplinar se torna propício já que, nessa fase, a gestante está aberta a adquirir informações e mudar seus comportamentos com intuito de melhorar sua saúde e a do bebê (Marla et al., 2018). Dito isso, fica evidente a viabilidade da realização do acompanhamento odontológico e a quebra de paradigmas por parte das gestantes, e dos profissionais de saúde, de que o pré-natal odontológico possa implicar em riscos à gestação (Botelho et al., 2019). Sendo assim, a instrução de uma alimentação materna adequada, rica em vitaminas indispensáveis para a formação dentária, os cuidados relacionados a saúde bucal da gestante e do bebê, e a importância do aleitamento materno devem ser amestrados durante essa etapa e continuados logo após o nascimento da criança (Fernandes et al., 2010).

O aleitamento materno possui um papel chave no desenvolvimento saudável da criança e seus benefícios são inúmeros. As experiências vividas na primeira infância são extremamente importantes para determinar o caráter de um indivíduo na idade adulta (Mozzaquatro et al., 2015). Durante a amamentação acontece um íntimo contato entre mãe e filho e o fortalecimento do elo afetivo, que é de suma necessidade para seu desenvolvimento psicoafetivo, e isso se deve ao fato de que as crianças amamentadas possuem uma predisposição a serem mais tranquilas e fáceis de socializar-se durante a infância (Passos \& Ribeiro, 2019). O processo de amamentação está diretamente relacionando com o desenvolvimento das funções do 
sistema estomatognático, como sucção, mastigação, deglutição, respiração e fonação, e influencia no crescimento dos ossos, musculatura e articulações da face. Além disso, o leite materno oferece benefícios nutricionais e imunológicos, devido a sua composição (Salone et al., 2013; Peres et al., 2018).

A Organização Mundial da Saúde (OMS) e o Ministério da Saúde preconizam o início da amamentação dentro de uma hora após o nascimento, o aleitamento materno exclusivo nos primeiros 6 meses de vida e a amamentação complementar até os 2 anos de idade (Catalá et al., 2017; Peres et al., 2018). O leite materno é a principal fonte de nutrientes para o recémnascido, e o tempo de duração do processo de amamentação está diretamente relacionando ao início precoce de sua estimulação, que se dá através dos movimentos mandibulares de sucção. Dito isso, é de extrema importância certificar previamente se existem dificuldades no processo de mamada (Alves et al., 2019).

O presente artigo propõe, através de uma análise da literatura existente, elucidar informações esclarecedoras sobre a importância e necessidade da amamentação e os reflexos da mesma no sistema estomatognático da criança, os seus benefícios na saúde geral do binômio mãe-bebê, e as desvantagens na ausência dessa prática, sendo o desmame precoce um empecilho na vida do indivíduo e consequentemente há uma instalação de hábitos deletérios, e com isso, a privação do aleitamento materno posteriormente pode trazer problemas como desenvolvimento de oclusopatias, alterações do crescimento ósseo, respiração bucal, e funções anormais da língua durante a deglutição, fonação e postura (Gisfrede et al., 2016).

\section{Metodologia}

Trata-se de uma revisão narrativa da literatura. Esse tipo de estudo do ponto de vista teórico ou conceitual realizam uma revisão ampla a fim de descrever e discutir o desenvolvimento ou o "estado da arte", contribuindo com o debate de determinadas temáticas (Rother,2007). A presente revisão de literatura foi realizada através de uma pesquisa bibliográfica utilizando as bases de dados eletrônicos Biblioteca Virtual em Saúde (BVS), PUBMED e SCIELO - Scientific Electronic Library Online. Foram selecionados artigos científicos publicados no período entre 2010 a 2020. Os descritores utilizados foram: "amamentação e sistema estomatognático", "odontologia e gestação", "amamentação e saúde bucal", "aleitamento materno', "amamentação e maloclusões", "odontopediatria e amamentação", "sucção e aleitamento" e seus correspondentes em inglês. Para seleção dos artigos, incluímos os trabalhos disponíveis na íntegra, na língua inglesa e portuguesa, em que o tema correspondia ao analisado nesta revisão, e com isso foram excluídos trabalhos por falta de clareza de ideias, artigos que não se encaixavam no tema proposto, opiniões de especialistas, editoriais e resumos ainda que publicados em revistas indexadas.

\section{Resultados e Discussão}

\subsection{Leite materno e o processo de amamentação}

O leite materno apresenta propriedades únicas e, por isso, é considerado a melhor e mais segura forma de nutrição para as crianças de zero a seis meses de idade. Os primeiros 1000 dias de vida é um momento oportuno para a formação da criança com consequências que irão refletir na vida adulta e é nessa fase que se constrói condições positivas ou negativas para o desenvolvimento físico, psíquico e emocional do indivíduo (Araújo et al., 2019). A Organização Mundial da Saúde (OMS) orienta que a amamentação deve ser iniciada aproximadamente uma hora após o nascimento e deve ser preconizado que ela continue de forma exclusiva até o sexto mês de vida, evitando assim o consumo precoce de açúcares na vida da criança e, após esse período uma dieta complementar pode ser inserida com o objetivo de suprir as necessidades nutricionais do bebê mantendo-a até os 24 meses de vida, alcançando assim os 1000 dias do bebê essenciais para o desenvolvimento (Peres et al., 2018; Araújo et al., 2019). 
A lactação é o resultado natural da gravidez e do parto, como uma parte integrante do processo reprodutivo que beneficia a mãe e filho simultaneamente. Embora seja extensamente conhecido que a amamentação é uma etapa importante no processo reprodutivo da mulher, e que a mesma acarreta inúmeros benefícios, as informações fornecidas no pré-natal, práticas de puericultura e atividades de conscientização muitas vezes negligenciam a repercussão positiva da amamentação na saúde da mãe. Nesse contexto, a literatura demonstra que a amamentação tem um impacto primordial não somente na saúde da criança, mas também na da mulher a curto, médio e longo prazo (Ciampo et al., 2018).

Para as crianças, o aleitamento materno diminui os riscos de desenvolverem otite média aguda; gastroenterite e diarreia; infecções do trato respiratório; enterocolite necrosante; leucemia; síndrome de morte súbita infantil (SIDS); asma; diabetes; colesterol alto; hipertensão; obesidade e patologias bucais(Salone et al., 2013). No que se diz a respeito à mulher, o ato de amamentar pode contribuir com a retomada do peso; menores chances de desenvolver sangramento e hemorragia pósparto; diabetes tipo 2; hipercolesterolemia; hipertensão e doença coronariana; obesidade; doença metabólica; osteoporose ; fratura de quadril; artrite reumatoide; depressão pós-parto; esclerose múltipla; endometriose e câncer de mama e de ovário (Salone et al., 2013; Brasil, 2015; Ciampo et al., 2018) . Sendo assim, a amamentação é uma estratégia eficiente para a prevenção a cada ano, de 823 mil mortes de crianças menores de cinco anos e de 20 mil mulheres por câncer de mama (Santos et al., 2016; Victoraet al., 2016).

O aleitamento materno é descrito quando a criança recebe o leite materno, seja direto da mama ou ordenhado. No entanto, esse processo pode ser desempenhado de diversas formas, como exemplos, o aleitamento materno predominante advém quando a criança além do leite materno ingere água ou líquidos à base de água, como chás, infusões e suco de fruta; o aleitamento materno misto ou parcial, quando a criança recebe o leite materno e outros tipos de leite; e o aleitamento materno complementado sucede quando a criança recebe qualquer tipo de leite (materno, fórmulas artificiais, ou outros) e também consome alimentos sólidos ou semissólidos, com a finalidade de complementar a amamentação e não de substituí-la . Por fim, o aleitamento materno exclusivo, sendo o mais indicado, é aquele que ocorre quando a criança recebe somente o leite materno, direto da mama ou ordenhado, ou leite humano proveniente de um banco de leite, sem que ocorra a ingestão de outros tipos de líquidos ou sólidos, com exceção de medicamentos (Brasil, 2015).

O posicionamento do bebê para uma pega apropriada permite o completo esvaziamento da mama, e como consequência aumenta a produção de leite além de prevenir contra o traumatismo e ingurgitamento mamário, introdução desnecessária de alimentos complementares e o desmame precoce (Souza et al., 2020). A configuração adequada da mãe facilita a disposição da boca do bebê em relação à aréola e mamilo. Dito isso, o bebê deve estar bem apoiado, com a cabeça e o corpo alinhado bem próximo e voltado para a mãe, barriga com barriga, o queixo tocando o peito e a boca bem aberta, de frente para o mamilo (Pires, 2012).

Para que a pega mamária correta aconteça é necessário que o bebê realize uma abertura ampla da boca, abocanhando o mamilo e a aréola de forma que ocorra um vedamento entre essas estruturas, formando um vácuo que será fundamental para que o mamilo e a aréola se mantenham dentro da cavidade oral da criança. Além disso, a língua deve assumir a posição de canolamento, na qual suas bordas laterais e a ponta formam uma concha. A remoção do leite, chamada de ordenha, se inicia através do movimento peristáltico rítmico da ponta da língua para trás que pressiona suavemente o mamilo, nesse momento o leite é levado até a faringe posterior e esôfago ativando o reflexo de deglutição (Brasil, 2015).

O primeiro leite secretado pela mãe, produzido desde o último trimestre da gestação até a primeira semana pós-parto é chamado de "colostro" e possui grande quantidade de água, se apresentando com um aspecto acinzentado similar à água de coco, dispondo de proteínas (mais albumina e globulina), menor conteúdo de lactose e gordura, maior concentração de sais minerais, fatores de crescimento e fatores imunológicos como, $\operatorname{IgM}$, $\operatorname{IgE}, \operatorname{IgD}$ e em maior predominância o IgA. Essas células são responsáveis por colonizar a mucosa gastrointestinal do neonato, que se apresenta isenta de defesa, impedindo a aderência 
e colonização de patógenos entéricos (Silva et al., 2017). Outra característica imunizante do leite materno é a presença de células polimorfonucleares como macrófagos, neutrófilos e eosinófilos encarregadas de fagocitar microrganismos insalubres, diminuindo no lactente a incidência e/ou a gravidade de patógenos (Brasil, 2015; Silva et al., 2017; Brasil, 2019).

Com o decorrer da mamada, entre o $7^{\circ}$ e o $14^{\circ}$ dia após o parto, o leite sofre variações e passa a ser chamado de "leite de transição", que se encontra com uma coloração branca opaca devido ao aumento dos níveis de caseína em sua composição e apresenta uma quantidade de gorduras e carboidratos maior, enquanto o teor de proteínas e minerais diminui gradativamente (Brasil, 2015; Silva et al., 2017; Brasil, 2019). A partir da terceira semana após o parto o leite é denominado "leite maduro", em virtude da dieta da mãe ele é mais amarelado, com maior concentração de betacaroteno, pigmento lipossolúvel presente na cenoura, abóbora e vegetais de cor laranja. Contém maiores quantidades de lipídeos e calorias, que são a principal fonte de energia para o crescimento e saciedade do lactente, e vitaminas que suprem as necessidades essenciais de vitaminas lipossolúveis e de ácidos graxos poliinsaturados (Brasil, 2015; Silva et al., 2017; Brasil, 2019).

\subsection{Sucção}

A sucção é um instinto natural e é a primeira atividade muscular coordenada do bebê, que desde o nascimento apresenta uma necessidade inerente a realizá-la e pode ser satisfeita de duas formas: através da sucção não-nutritiva e sucção nutritiva. A suçãao não-nutritiva pode ser observada através de hábitos como a sucção digital, de lábio, chupeta ou outros objetos e proporciona à criança prazer, sensação de bem-estar e proteção, satisfazendo-a psicologicamente. A sucção nutritiva ocorre através do aleitamento natural ou artificial, e é capaz de oferecer benefícios gerais, imunológicos, nutricionais e orais para a criança (Albuquerque et al., 2010; Ling et al., 2018).

O mecanismo de sucção se inicia através de quatro movimentos mandibulares, sendo eles: abertura; protrusão; fechamento; e retrusão mandibular. Para cumprir esse desígnio, o recém-nascido deve apresentar coordenação entre a sucçãodeglutição-respiração e uma boa estabilidade e mobilidade motora (Alves et al., 2019). O aleitamento natural, realizado por meio da sucção, promove um intenso exercício da musculatura orofacial, essa ação repetitiva aumenta o tônus muscular e promove o desenvolvimento ósseo e muscular, além disso, gera fadiga nos músculos, fazendo com que a criança satisfaça seu instinto de sugar e não necessite de uma sucção não nutritiva (Moimaz et al., 2013).

Os hábitos orais são encarados como normais ou deletérios. A respiração nasal, a mastigação e a deglutição são classificadas como hábitos fisiológicos e funcionais, já que auxiliam no desenvolvimento orofacial em toda sua plenitude e sem desequilíbrio. No entanto, a respiração bucal, sucção digital, de chupeta, de mamadeira ou outros objetos, são considerados hábitos não fisiológicos, ou seja, hábitos deletérios (Boeck et al., 2013).

O desmame precoce pode favorecer o aparecimento de hábitos orais não fisiológicos, como a sucção não nutritiva. Isso ocorre, pois, a musculatura perioral não é tão estimulada como acontece na sucção do leite materno, sendo assim, a criança tende a buscar outro tipo de sucção, como dedo, lábio ou chupeta a fim de se satisfazer nutricional e/ ou emocionalmente (Miotto et al., 2014; Neu et al., 2014).

Crianças com menor tempo de aleitamento materno exclusivo possuem sete vezes maior risco de adquirir hábitos de sucção nocivos quando comparadas com as crianças aleitadas no seio materno pelo período correto. Quando essa prática ocorre após os três anos com uma frequência e intensidade maior, esse hábito se torna um possível fator etiológico para o desenvolvimento de oclusopatias, alterações do crescimento ósseo, respiração bucal e funções anormais da língua durante a deglutição, fonação e postura (Moimaz et al., 2013; Miotto et al., 2014).

A literatura demonstra que a duração dos hábitos de suç̧ão nocivos exercidos pela criança pode gerar modificações na arcada dentária e outro prejuízo bastante comum seria relacionado ao desmame precoce que pode ocorrer, pois existe uma confusão dos bicos, sendo o bico da chupeta e da mamadeira diferentes, dessa forma, na hora da sucção no seio materno, pode 
existir uma dificuldade e se obter o desmame devido a isso. Então, logo que os hábitos são interrompidos entre os 24 e os 36 meses, há um risco aumentado de desenvolver mordida cruzada e aumento da distância intercanina mandibular, quando comparado com os hábitos que são suspensos aos 12 meses. Enquanto a interrupção entre os 36 e 48 meses contrasta maior prevalência de overjet aumentado, mordida aberta e maior profundidade maxilar em comparação com a paralisação do hábito mais precocemente (Passos \& Frias-Bulhosa, 2010; da Silva,2021).

\subsection{Sistema estomatognático}

O sistema estomatognático é constituído por estruturas integrantes do sistema digestório e respiratório, responsáveis por executar funções como sucção, deglutição, mastigação, fala e respiração. A amamentação está diretamente relacionada com o desenvolvimento desse sistema, sendo um artifício de prevenção para que as suas funções sejam efetuadas de forma habitual (Pacheco et al., 2012; Gisfrede et al., 2016).

Em condições fisiológicas a respiração é desempenhada por via nasal visto que, a cavidade nasal tem a competência de purificar o ar fazendo com que ele chegue aos pulmões em temperatura ideal, evitando a inserção de microrganismos que não são fundamentais ao organismo e dessa forma, protegendo as vias aéreas inferiores. Além disso, respiração nasal propicia o adequado crescimento maxilar e a postura exata da mandíbula, que viabiliza a posição dos lábios, língua, bochechas e intercuspidação entre arcadas dentárias se encontrem de forma correta (Felcar et al., 2010; Pacheco et al., 2012).

No entanto, as crianças podem desenvolver a respiração bucal, que se manifesta devido uma etiologia multifatorial, sendo por uma predisposição anatômica; por obstrução das vias aéreas superiores, no caso em que o indivíduo apresenta hipertrofia das tonsilas palatinas, adenoides, desvio de septo nasal, pólipos nasais, rinites alérgicas e não alérgicas, sinusites, hipertrofias de cornetos, aleitamento artificial; e, principalmente frente a presença de um hábito oral nocivo, tais como suç̧ão digital, labial ou de chupeta que dependendo da intensidade, frequência e duração, fazem com que a passagem do ar seja feita pela boca. Esta disfunção respiratória modifica o padrão de crescimento da face ocasionando também, em alguns casos, alterações morfofuncionais em todo o organismo como cianose infraorbitária, apneia do sono, hipoventilação, o tórax pode se desenvolver de forma atípica, deglutição atípica e face alongada (Felcar et al., 2010;Passos\&Frias-Bulhosa, 2010;Johanns et al., 2011; Pacheco et al., 2012; Gisfrede et al., 2016). Predispondo também o desenvolvimento futuro, em alguns casos, de bruxismo, de mordida cruzada; mordida aberta anterior; língua hipotônica, repousando no assoalho bucal; língua baixa; palato ogival; apinhamento dentário; alto índice de cárie; lábios evertidos; bochechas flácidas; mandíbula que se mantém aberta e a língua apresentando um volume maior do que o normal (Gisfrede et al., 2016; Cassimiro et al., 2019).

Outra condição singular é a deglutição atípica, que é descrita como a incapacidade do indivíduo de ingerir e transportar os nutrientes vitais ao corpo humano de forma satisfatória. Os indivíduos portadores dessa desordem apresentam lábios, língua, bochechas e músculos elevadores da mandíbula hipotônicos com os seguintes aspectos: lábios evertidos, bochechas flácidas, mandíbula se mantém aberta e a língua apresenta um volume maior do que o normal. A deglutição fisiológica ocorre através da contração ativa dos músculos dos lábios, bochechas e língua. Uma vez que esses músculos se apresentam hipotônicos, a ponta da língua se posiciona de forma mais anterior entrando em contato com o lábio inferior, provocando uma limitação da atividade da parte mais posterior da língua e dos músculos da faringe, fazendo com que o processo de deglutição ocorra de forma anormal (Passos \& Frias-Bulhosa, 2010; Gisfrede et al., 2016).

\section{Conclusão}

Dado o exposto, torna-se imprescindível a realização do aleitamento materno natural e exclusivo durante os seis primeiros meses do recém-nascido, e após este período, uma dieta complementar pode ser inserida mantendo a amamentação até 24 meses de vida. Visto que a amamentação continua a ser o método mais indicado e seguro de nutrição para o recém- 
nascido, além de propiciar benefícios na saúde em geral a curto, médio e longo prazo na vida do binômio mãe-bebê.

Essa medida será um método simples e eficiente para prevenir que a criança adquira hábitos orais nocivos, sejam eles suç̧ão digital, labial, chupeta ou de outros objetos. Esses hábitos, quando se dão em alta intensidade, frequência e duração modificam o padrão de crescimento, afetando diretamente o desenvolvimento harmônico do sistema estomatognático.

\section{Referências}

Albuquerque, S. S. L. D., Duarte, R. C., Cavalcanti, A. L., \& Beltrão, É. D. M. (2010). A influência do padrão de aleitamento no desenvolvimento de hábitos de sucção não nutritivos na primeira infância. Ciência\&SaúdeColetiva, 15, 371-378.

Alves, Y. V. T., Santos, J. C. D. J., Barreto, I. D. D. C., Fujinaga, C. I., \& Medeiros, A. M. C. (2019). Full term newborns in non-nutritive suction evaluationand their relation on feeding performance. Revista Brasileira de Saúde Materno Infantil, 19(3), 621-630.

Araújo, H. R. V., de Carvalho, M. T., Imparato, J. C. P., \&Pinchemel, E. N. B. (2019). A importância do aleitamento materno no controle do desenvolvimento de hábitos deletérios: Revisão de Literatura/The importance of breastfeeding in controllingthedevelopment of harmfulhabits: A Literature Review. ID online Revista de psicologia, 13(47), 1135-1144.

Bendo, C. B., Martins, C. C., Pordeus, I. A., \& Paiva, S. M. D. (2014). Impacto das condições bucais na qualidade de vida dos indivíduos. Revista da Associacao Paulista de Cirurgioes Dentistas, 68(3), 189-193.

Boeck, E. M., Pizzol, K. E. D. C., Barbosa, E. G. P., Pires, N. C. D. A., \&Lunardi, N. (2013). Prevalência de má oclusão em crianças de 3 a 6 anos portadoras de hábito de sucção de dedo e/ou chupeta. Revista de Odontologia da UNESP, 42, 110-116.

Boronat-Catalá, M., Montiel-Company, J. M., Bellot-Arcís, C., Almerich-Silla, J. M., \&Catalá-Pizarro, M. (2017). Association between duration of breastfeeding and malocclusions in primary and mixed dentition: A systematic review and meta-analysis. Scientificreports, 7(1), 1-11.

Botelho, D. L. L., Lima, V. G. A., Barros, M. M. A. F., \& de Sousa Almeida, J. R. (2019). Odontologia e gestação: a importância do pré-natal odontológico. SANARE-Revista de Políticas Públicas, 18(2).

Brasil. (2011). Atenção à Saúde do Recém-Nascido Guia para os Profissionais de Saúde: Cuidados gerais. Ministério da Saúde.

Brasil. (2019). Manual de Normas e Rotinas de Aleitamento Materno. Ministério da Educação.

Brasil. (2015). Saúde da criança: aleitamento materno e alimentação complementar. Ministério da Saúde.

Cassimiro, I. G. V., de Souza, P. G., Rodrigues, M. C., \& Carneiro, G. K. M. (2019). A importância da amamentação natural para o sistema estomatognático. Revista uningá, 56(S5), 54-66.

Ciampo, L.A.D., Ciampo, I. R. L D. Aleitamento materno e seus benefícios para a saúde da mulher. Revista Brasileira de Ginecologia e Obstetrícia, v. 40, n. 6, p. 354-359, 2018.

da Silva, J. N. (2020). Aleitamento materno: motivos e consequências do desmame precoce em crianças. Revista Artigos. Com, 20 , e4756-e4756.

Felcar, J. M., Bueno, I. R., Massan, A. C. S., Torezan, R. P., \& Cardoso, J. R. (2010). Prevalence of mouth breathing in children from an elementary school. Ciencia\&saudecoletiva, 15(2), 427-435.

Fernandes, D. S. C., Klein, G. V., de Oliveira Lippert, A., de Medeiros, N. G., \& de Oliveira, R. P. (2010). Motivo do atendimento odontológico na primeira infância. Stomatos, 16(30), 4-10.

Gisfrede, T. F., Kimura, J. S., Reyes, A., Bassi, J., Drugowick, R., Matos, R., \& Tedesco, T. K. (2016). Hábitos bucais deletérios e suas consequências em Odontopediatria. Revista Brasileira de Odontologia, 73(2), 144.

Johanns, C. M., Silvério, K., Furkim, A. M., \&Marchesan, I. (2011). Is there a relation between harmful oral habits and facial typology and dental occlusion? Revista CEFAC, 13(6), 1095-1102.

Ling, H. T. B., Sum, F. H. K. M. H., Zhang, L., Yeung, C. P. W., Li, K. Y., Wong, H. M., \& Yang, Y. (2018). The association between nutritive, non-nutritive sucking habits and primary dental occlusion. BMC oral health, 18(1), 1-10.

Marla, $\mathrm{V}$ et al. The importance of oral health during pregnancy: A review. MedicalExpress, 5.

Miotto, M. H. M. D. B., Caxias, F. P., Campos, D. M. K. D. S., Ferreira, L. D. F. P. E., \& Barcellos, L. A. (2014). Aleitamento materno como fator de proteção contra a instalação de hábitos bucais deletérios. Revista CEFAC, 16(1), 244-251.

Moimaz, S. A. S., Rocha, N. B. D., Garbin, A. J. I., \&Saliba, O. (2013). A influência da prática do aleitamento materno na aquisição de hábitos de sucção não nutritivos e prevenção de oclusopatias. Revista de Odontologia da UNESP, 42, 31-36.

Mozzaquatro, C. O. de., Arpini, D. M., \& Polli, R. G. (2015). Relação mãe-bebê e promoção de saúde no desenvolvimento infantil.Psicologia em Revista, Belo Horizonte, 21(2), 334 a 351.

Neu, A. P., Silva, A. M. T. D., Mezzomo, C. L., \&Busanello-Stella, A. R. (2014). Aleitamento: Relação com hábitos de sucção e aspectos socioeconômicos familiares. Revista CEFAC, 16, 883-891. 
Research, Society and Development, v. 10, n. 14, e211101421988, 2021

(CC BY 4.0) | ISSN 2525-3409 | DOI: http://dx.doi.org/10.33448/rsd-v10i14.21988

Peres, K. G., Chaffee, B. W., Feldens, C. A., Flores-Mir, C., Moynihan, P., \& Rugg-Gunn, A. (2018). Breastfeeding and oral health: evidence and methodological challenges. Journal of dental research, 97(3), 251-258.

Pires, M. M. S., Obelar, M. S., Wayhs, M. L. C., \& Brasil, A. L. D. (2012). Manual de orientação do departamento de nutrologia: alimentação do lactente, do pré-escolar, do escolar, do adolescente e na escola. (3a ed.). SociedadeBrasileira de Pediatria, 41.

Rother, Edna TerezinhaRevisão sistemática X revisão narrativa. (2007). Acta Paulista de Enfermagem. 20 (2), v-vi. <https://doi.org/10.1590/S010321002007000200001>. https://doi.org/10.1590/S0103-21002007000200001.

Salone, L. R., Vann Jr, W. F., \& Dee, D. L. (2013). Breastfeeding: an overview of oral and general health benefits. The Journal of the American Dental Association, 144(2), 143-151.

Santos, B. Z., Dotto, P. P., \& Guedes, R. S. (2016). Breastfeeding and risk of dental caries. Epidemiologia e Servicos de Saúde, 25, 633-635.

Silva, D., Soares, P., \& Macedo, M. V. (2017). Aleitamento materno: causas e consequências do desmame precoce. UnimontesCientífica, 19(2), 146-157.

Souza, T. O. D., Morais, T. E. D. V., Martins, C. D. C., Bessa, J. D., \& Vieira, G. O. (2020). Efeito de uma intervenção educativa sobre a técnica de amamentação na prevalência do aleitamento materno exclusivo. RevistaBrasileira de SaúdeMaternoInfantil, 20, 297-304.

Pacheco, A. D. B., Silva, A. M. T. D., Mezzomo, C. L., Berwig, L. C., \& Neu, A. P. (2012). Relação da respiração oral e hábitos de suç̧ão não-nutritiva com alterações do sistema estomatognático. Revista Cefac, 14, 281-289.

Passos, I. V. D. C., \& Ribeiro, D. C. (2019). Aleitamento materno e sua influência na vinculação entre mãe-bebê.Anais do I e do II seminário de produção científica do curso de psicologia da Unievangélica, 1-18.

Passos, M. M., \& Frias-Bulhosa, J. (2010). Hábitos de sucção não nutritivos, respiração bucal, deglutição atípica-Impactos na oclusão dentária. Revista Portuguesa de Estomatologia, MedicinaDentária e CirurgiaMaxilofacial, 51(2), 121-127.

Victora, C. G., Barros, A. J., França, G. V., Bahl, R., Rollins, N. C., Horton, S., \& Walker, N. (2016). Amamentação no século 21: epidemiologia, mecanismos, e efeitos ao longo da vida. Epidemiol Serv Saúde, 25(1), 1-24. 